\title{
Harnessing the
} photochemistry of bromine for sustainable manufacturing

Chemical production can be fraught with hazards. From explosions to toxic chemicals, making useful molecules often requires careful thought about how to make a process inherently safer. Researchers at an (Anstra) have developed an ingenious way to use
automated flow reactors manufactured by Corning SAS (France) to safely harness the photochemistry of bromine a highly toxic, sludge-brown substance that can be used to make some of the most useful chemical bonds for
pharmaceutical production.

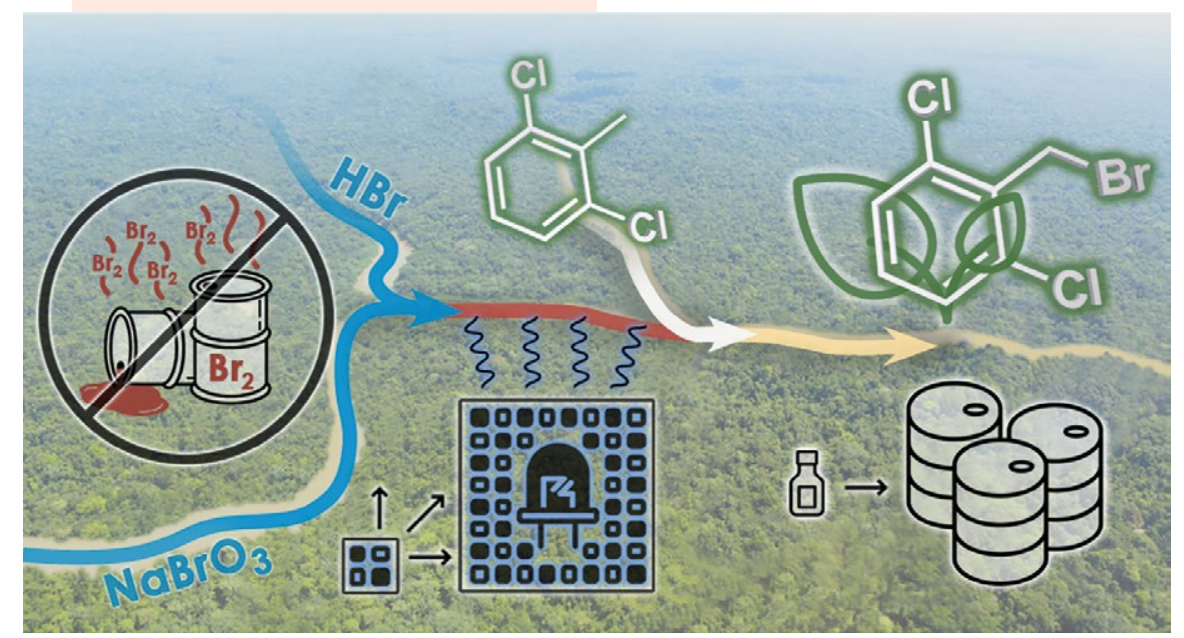

Hydrogen bromide (HBr) and sodium bromate $\left(\mathrm{NaBrO} \mathrm{O}_{3}\right)$ are dissolved in water, so they don't mix
well with the oil-like reactant. Constant mixing through the reactor allows the reaction to occur. production. Another is flow chemistry, where the chemical reagents are constantly mixed, reacting and producing new chemical species.

Flow reactors can be a brilliant way to produce high yields of chemicals, particularly for special types of reactions, by light (known as photochemical reactions). However, both flow ral and photochemistry have not been widely adopted by the pharmaceutical industry due to the challenges of finding methods to scale up chemical reactions to produce the large quantities of products needed. This has meant that, to date, most reactions that use these technologies and methods have been limited to lab scale.

To remedy this, researchers at the Research Center Pharmaceutical Engineering (RCPE) in Austria, in collaboration with Corning ${ }^{\circledR}$ AdvancedHow'M Reactors, France, have designed a highly optimised reaction procedure using a flow reactor for light-triggered reactio
that can be scaled up to produce industrial quantities. This process, which runs in a reactor roughly the size of an A4 piece of paper, is highly efficient: if left to run continuously, it is conceivable that it may meet a significant portion of the world's demands for a particular

CHEMISTRY WITH LIGHT Light is a powerful tool for chemistry, exploited both by nature and by scientists in the lab. Photochemical reactions, or reactions triggered by light, are at the heart of photosynthesis, the process that converts solar energy to plant food and oxygen for us to breath

What makes light a powerful chemica reagent is the energy it can provide.

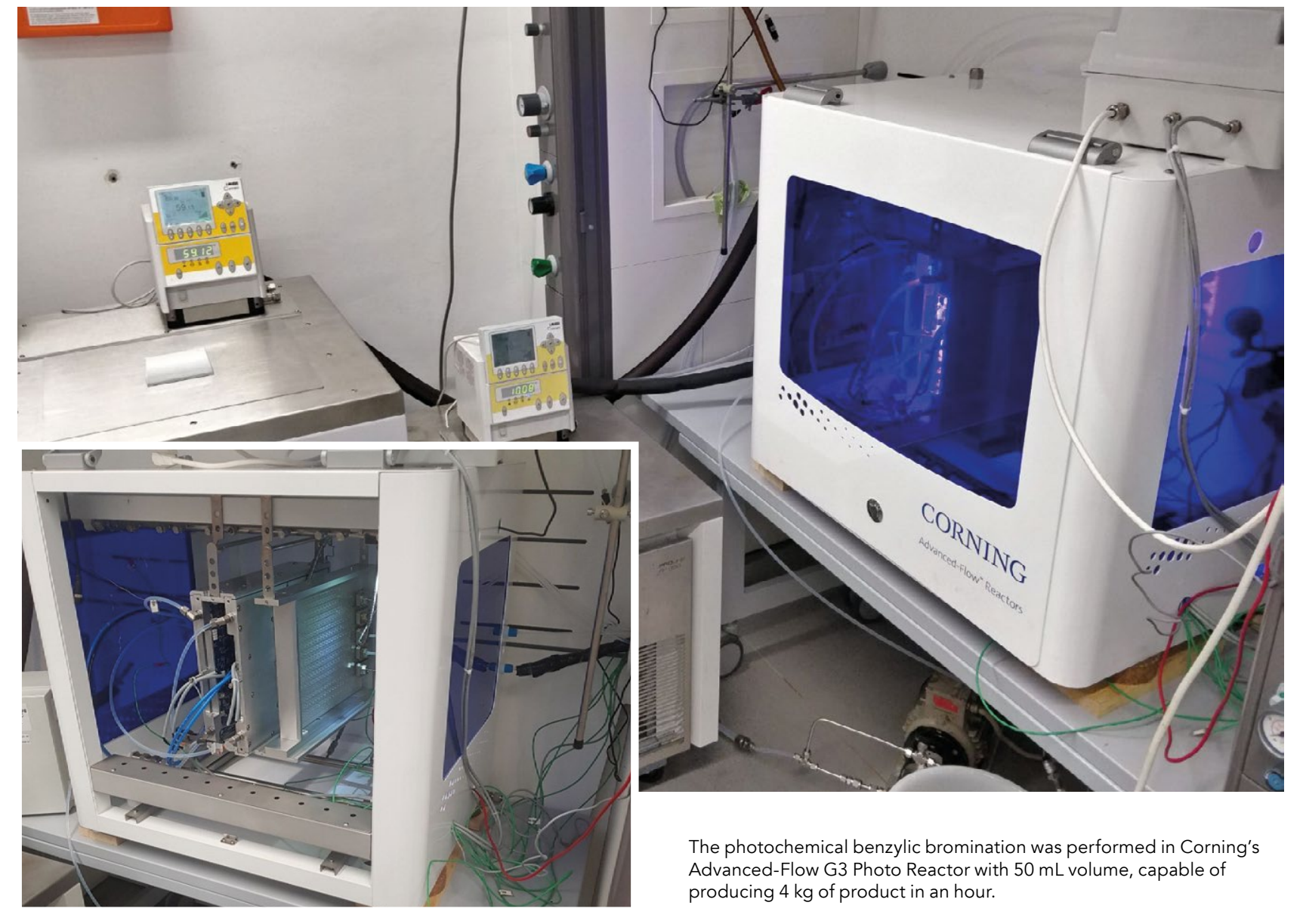

Light is a renewable energy source and, unlike concentrated acids and alkalis, does not generate waste that needs to be dealt with afterwards.

Rather than vigorously heating a molecule make it react, absorbing a single packet of light energy known as a photon light-driven chemistry of a molecule can be very different to what happens when it is heated, opening many new exciting reaction possibilities.

Researchers at RCPE and Corning SAS are also excited by the environmentally friendly possibilities offered by photochemical reactions. Light is a renewable energy source and, unlike concentrated acids and alkalis, does not generate waste that needs to be dealt with afterwards. It is safe to hand and often allows for 'gentler' reaction conditions that are not so energy intensive as needing to heat large vats With the advent of powerful, reliable light-emitting diodes (LEDs), industrial than ever. Corning's range of flow photoreactors incorporate these LEDs in a highly engineered manner, to create platform for synthesising many chemica products in an efficient way. Scaling up photochemical synthesis to industrial levels poses some unique challenges: as the reactors get bigger, it is harder for the light to reach the middle of the vesse and trigger the desired chemistry in the whole batch.

A significant portion of the work done by RCPE was finding optimal reactor setup and conditions that would not just facilitate photochemistry in the lab scale. With the combination of carefully they have been able to achieve several remarkable things for a target reaction: eliminating the need for organic solvents, recycling the acidic reagent reducing waste and increasing safety, al

\section{RADICAL BROMINE}

The reaction focused on in this study involved a famously hazardous chemical reagent, bromine $\left(\mathrm{Br}_{2}\right)$. While formally liquid at room temperature, it

readily evaporates to form an ominous brownish-red gas. While not as toxic as its more famous chemical counterpart chlorine, it is still very harmful to human health and lethal in reasonably small doses. To trigger carbon-bromine bond formation in synthesis, light is also added to the mix to trigger the bromine radicals

Bromine is a chemist's best friend in the synthesis of many pharmaceutical drugs This has prompts and advanced materials. tuned chemistry and a suitable reactor, breakage of the bromine-bromine 


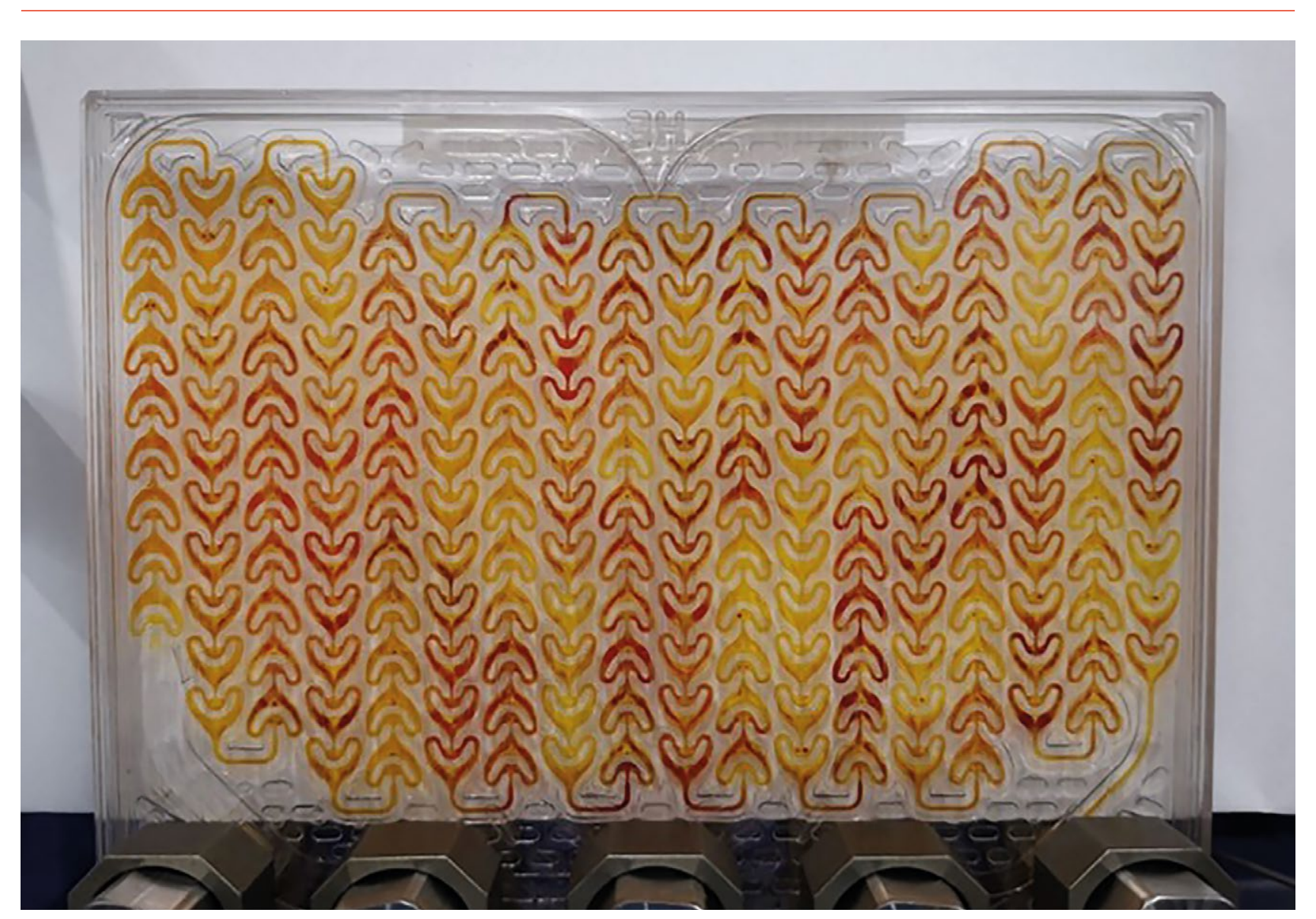

The researchers stilised Cornin's' heart-shaped fluidic module design that enables very high flow rates to create a highly turbulent environment where
bromine and the starting material can be mixed.

This photochemical flow procedure is an important demonstration of how photochemical reactions can be scaled up to industrial levels.

utilise bromine, whilst avoiding transport and storage of the reagent itself. Rather than adding toxic bromine to their flow developed a system to generate the needed bromine in situ. The bromine is formed continuously as it is needed. This means all the bromine is consumed in the chemical reaction and no harmful reagents are left over.

\section{HEART-SHAPED SCIENC}

Getting the reagent, the reaction for bromine, and adding the light all at the right time in the right amounts is a tricky balancing act. To make the reaction occur, the starting material and bromine need to be mixed very well. As they are water, this is rather difficult. capable of producing $4 \mathrm{~kg}$ of product in an hour. Their approach is a new and exciting way of doing old chemistry efficiently, effectively and in a more environmentally friendly fashion. This was also a breakthrough in demonstrating the viability of taking the reaction optimisation for photochemical flow reactors from lab large-scale manufacturing.

To do this, the team utilised Corning's highly engineered heart-shaped fluidic module design that enables very high chemicals can meet. Using powerfu LEDs means that precisely as all the reagents are mixed, the breakage of the bromine bond can be triggered, and the their initial tests on a small scale with a $3 \mathrm{~mL}$ reactor and spent many months tuning parameters, such as the reaction temperatures and pressures to find the optimal conditions for maximising methodology that worked, the RCPE researchers, with the help of Microinnov $\mathrm{GmbH}$, could relatively quickly scale up Having alternative synthesis tools, which environment where all the necessary final products formed. The team started product yield. Once they had found
As well as proving a highly efficient route to manufacture specific drug intermediates in an inherently safe and automated way, this photochemical flow of how photochemical reactions can be scaled up to industrial levels - something that has proved very difficult in the past. open new chemistry while being more efficient, is an exciting prospect for the have demonstrated how the combination of How and photochemistry is an excellent option for pharmaceutical manufacture on
the industrial scale. procedure is an important demonstration environmentally conscious and energy future of industrial synthesis and the team

\section{Behind the Research} 固

Dr Jason Williams

E: jason.williams@rcpe.at W: http://goflow.at

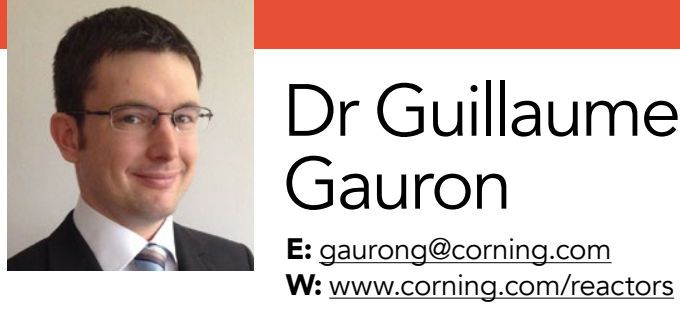

Research Objectives

Utilising in situ bromine generation, researchers at RCPE have developed photochemical benzylic bromination from lab to pilot scale in Corning ${ }^{\circledR}$ Advanced-Flow'M Reactors.

\section{Detail}

Jason Williams
Research Center Pharmaceutical

Engineering $\mathrm{GmbH}$

Inffeldgass

Guillaume Gauron

Corning SAS

Reactor Technologies

7 bis, Avenue de Valvins

77210 AVON Cedex, France

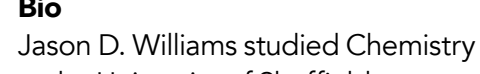
at the University of Sheffield,

before undertaking a PhD on the

GlaxoSmithKline/University or Strath

Prof William Kerr. Since 2018, Jason has worked with Prof C. Oliver Kappe in the CCFLOW consortium, focusing on flow photochemistry and real-time reaction analysis.

After earning his $\mathrm{PhD}$ in Organic Chemistry from Orsay University Paris $X$ R\&D radiochemist at IBA Molecular (Cis Bio) from 2008 to 2014 before joining Corning Reactor Technologies as an Application Engineer. In 2018, Guillaume accepted the role of EMEA Technical Sales Manager.

Funding

CC FLOW Project (Austrian Research

Promotion Agenject (Austrian Rese funded through the Austrian COMET Program by the Austrian Federal Ministry of Transport, Innovation and Technology (BMST), the Austrian Federal Ministy (BMWFW), and by the State of Styria (Styrian Funding Agency SFG).

\section{Collaborators}

- Alexander Steiner, C. Oliver - Philippe Roth, Marc Winter (Corning Incorporated) - Oscar De Frutos, Juan Rincon Carlos Matteos (Eli Lilly) Franz Strauss, Gunter Tekau (Microinnova)
References

Steiner, A., Williams, J.D., de Frutos, O., Rincon, J.A., Mateos, C., and Kappe, C.O. (2020). Continuous photochemical benzylic bromination using in situ generated $\mathrm{Br}_{2}$ : process intensification towards optinal $P$ Mand throughput. Green Chemistry, 22, 488 ggc03662

Steiner, A., Roth, P.M.C., Strauss, F.J., Gauron, G., Tekautz, G., Winter, M. Williams, J.D., and Kappe, C.O. (2020). Multikilogram per Hour Continuous Photochemical Benzylic Brominations Applying a Smart Dimensioning Scale-up Available at: https://doi.org/10.1021/acs.oprd.0c00239

\section{$\Gamma$}

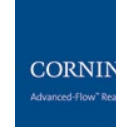

CCFLOW \}, क्री

\section{Personal Response}

What are the challenges to overcome so photochemistry can be more widely used in chemical synthesis?

When shining light on a reactor, this light will often all be absorbed within a few millimetres. This means that doing photochemical reactions in large reactors, as is usua forchemical manufacturing, is very challenging, since to reactions performed in the development lab. We use flow chemistry to overcome this challenge by flowing millimetre depth, meaning that it can all absorb the same amount of light. This makes things easier, but we still need to change reactor dimensions to make more material, which
remains a challenge. 